\title{
Aneurismas y divertículos cardíacos congénitos: reporte de una serie pediátrica
}

\author{
Patricia Álvarez ${ }^{a b c}$, Patricia Aroca ${ }^{d}$, Valeria Acevedo ${ }^{a b c}$, Patricia Ríos $^{e}$, Rosa Arellano ${ }^{f}$. \\ a Cardióloga Pediatra, Hospital Roberto del Río, Santiago. Chile. \\ b Departamento de Pediatría Norte, Facultad de Medicina, Universidad de Chile, \\ c Clínica Alemana de Santiago. Chile. \\ d Programa de formación en Cardiología Pediátrica, Universidad de Chile. \\ e Interna de Medicina. Facultad de Medicina, Universidad de Chile. \\ f Cardióloga Pediatra, Hospital Carlos Van Buren, Valparaíso. Chile. \\ * No hubo apoyo financiero.
}

Introducción: Los aneurismas y divertículos cardíacos congénitos constituyen malformaciones poco frecuentes y generalmente comprometen el ventrículo izquierdo. Pueden presentarse en forma asintomática, causar embolización, arritmia, insuficiencia cardíaca, rotura o muerte súbita. La ecocardiografía Doppler color realiza el diagnóstico y detecta malformaciones asociadas. La terapia puede ser médica o quirúrgica dependiendo de la presentación clínica y de los hallazgos en cada paciente.

Método: Estudio retrospectivo de 5 pacientes durante un período de diez años, uno con divertículo, cuatro con aneurismas, en los cuales se analizó sexo, edad, motivo de consulta, sintomatología, hallazgos al examen físico, malformaciones extracardíacas o cardíacas asociadas. La ecocardiografía confirmó el diagnóstico y analizó la localización, efecto sobre la función cardíaca y presencia de complicaciones. Se estudió el tipo de tratamiento elegido como terapia antiplaquetaria, antiarrítmica o quirúrgica, además del seguimiento.

Resultados: De un total de 5 pacientes, tres eran hombres, la mediana de edad al diagnóstico fue de 13 meses; dos de diagnóstico antenatal. Soplo cardíaco (3/5) fue el principal hecho que motivó el estudio. La ubicación más frecuente fue en el ventrículo izquierdo y la malformación cardíaca asociada la comunicación interventricular (2 pacientes). La terapia elegida fue médica en todos los pacientes con aspirina y en un caso se agregó atenolol por taquicardia ventricular no sostenida. Ningún paciente falleció.

Conclusión: Constituye la primera publicación sobre esta patología en nuestro país; si bien es una serie pequeña, aporta aspectos clínicos, diagnósticos y de seguimiento que ayudan a sospechar esta rara malformación en el paciente pediátrico.

\section{Correspondencia:}

Dra. Patricia Álvarez Zenteno.

pazenteno@yahoo.com.

Fono contacto: +569-98795725 


\section{Congenital cardiac aneurysms and diverticula: a pediatric series}

Introduction: Congenital cardiac aneurysms and diverticula are rare cardiac malformations which mainly affects the left ventricle. Clinically, most are asymptomatic or may cause systemic embolization, arrhythmia, heart failure, ventricular wall rupture or sudden cardiac death. Doppler echocardiography establishes the diagnosis and can detect associated malformations. Treatment may be medical or surgical, depending on findings.

Patients and Methods: Retrospective study of 5 patients followed for ten years. One patient presented with a diverticulum and four with aneurysms. We analyzed sex, age at diagnosis, symptoms, physical examination, and associated cardiac and extracardiac malformations. Echocardiography confirmed the diagnosis and established location, cardiac function, and presence of complications. We analyzed the treatments used: antiplatelet, anti-arrhythmic or surgical therapy, and follow up.

Results: Three were males, the median age at diagnosis was 13 months; in two patients the diagnosis was made before birth. Heart murmur in 3 of 5 patients was the main presenting finding. The most frequent location was the left ventricle and the associated cardiac malformation was ventricular septal defect in 2 patients. All patients were treated with aspirin. One patient with non-sustained ventricular tachycardia received atenolol. There were no deaths.

Conclusion: This is the first report of this disease in our country. Although it is a small series it provides important clinical aspects for the diagnosis, treatment and follow-up that help us suspect this rare malformation in pediatric patients.

Key words: Diverticula, aneurysm, congenital cardiac disease. 


\section{Introducción}

Los aneurismas y divertículos cardíacos congénitos constituyen malformaciones poco frecuentes, que se manifiestan como una protrusión localizada en la pared cardíaca, generalmente del ventrículo izquierdo, pero pueden originarse en cualquier otra cavidad ${ }^{1}$.

Los aneurismas cardíacos se caracterizan por tener paredes de tejido fibroso con escasas fibras miocárdicas, lo que aparece como zonas aquinéticas o disquinéticas. Poseen una boca amplia hacia la cavidad ventricular y se presentan, en general, como defectos cardíacos aislados. Los divertículos, en cambio, conservan la función contráctil, al estar formados por las tres capas del corazón; presentan una base estrecha de unión con la cavidad y se asocian frecuentemente con defectos de la línea media toraco abdominal ${ }^{2}$.

Se desconoce la etiología de estas malformaciones, atribuyéndola a una detención parcial en el desarrollo embrionario normal de la pared ventriculaa partir de la $4^{\mathrm{a}}$ semana de gestación. Su forma de presentación es variable, pudiendo ser asintomático o presentar arritmias, insuficiencia cardíaca e incluso muerte por embolia o rotura. El objetivo de este trabajo fue describir los aspectos clínicos, diagnósticos, y terapéuticos de una serie de 5 casos pediátricos controlados en un centro cardiológico único en un período de diez años.

\section{Pacientes y método}

\section{Diseño del estudio}

Estudio de cohorte, retrospectivo, de 5 pacientes porta- dores de divertículo o aneurisma cardíaco diagnosticados por ecocardiograma Doppler color, y tratados en el Servicio de Cardiología del Hospital de niños Roberto del Río, entre los años 2005 y 2015.

\section{Variables analizadas}

Se analizó edad, sexo, motivo de consulta, síntomas, examen físico y la presencia de malformaciones asociadas.

De los estudios de laboratorio se evaluó el Electrocardiograma (ECG) de 12 derivaciones en reposo, Holter de arritmias de 24 horas, Radiografía de Tórax y Ecocardiograma Doppler color y 3D. La ecocardiografía determinó la localización y el tipo de malformación, las características de la pared y su contractibilidad, tamaño de la boca, efecto sobre la función cardíaca y presencia de complicaciones como derrame pericárdico o trombos. Se revisó la Resonancia magnética cardíaca (RMC) en tres pacientes. La función ventricular fue estimada por la fracción de eyección según el método de Simpson. Se evaluó el tipo de tratamiento: terapia antiplaquetaria, antiarrítmica o quirúrgica; la evolución, aparición de complicaciones y el tiempo de seguimiento.

\section{Resultados}

Durante un período de 10 años, cuatro pacientes fueron tratados por aneurismas y uno por divertículo. La edad promedio al diagnóstico fue de 13 meses, con rango desde el período fetal a 3 años. Tres de ellos fueron hombres. Todos los pacientes eran asintomáticos. La forma de pre-

Tabla 1. Características de los pacientes, tipo de malformación y exámenes de laboratorio al momento del diagnóstico.

\begin{tabular}{|c|c|c|c|c|c|c|}
\hline $\mathrm{N}^{\circ}$ Caso & $\begin{array}{c}\text { Tipo de } \\
\text { Malformación }\end{array}$ & $\begin{array}{l}\text { Edad al } \\
\text { Diagnóstico }\end{array}$ & Sexo & $\begin{array}{r}\text { Radiografía } \\
\text { de Tórax }\end{array}$ & ECG & $\begin{array}{c}\text { Holter } \\
\text { de Arritmias }\end{array}$ \\
\hline 1 & D del VD & Fetal & M & Normal & Normal & Normal \\
\hline 2 & A del Septum & Fetal & M & $\begin{array}{l}\text { Silueta cardíaca } \\
\text { prominente }\end{array}$ & $\begin{array}{c}\text { Trastorno inespecífico } \\
\text { de la conducción } \\
\text { intraventricular }\end{array}$ & EV \\
\hline 3 & A del VI & 9 meses & $\mathrm{F}$ & Normal & Normal & Normal \\
\hline 4 & A del Septum & 2 años & $M$ & $\begin{array}{c}\text { Silueta cardíaca } \\
\text { prominente }\end{array}$ & Normal & Normal \\
\hline 5 & A del VI & 3 años & $\mathrm{F}$ & $\begin{array}{c}\text { Imagen redondeada } \\
\text { en ápex }\end{array}$ & $\begin{array}{l}\text { Trastorno inespecífico de la } \\
\text { conducción intraventricular }\end{array}$ & Normal \\
\hline
\end{tabular}

D: divertículo; VD: ventrículo derecho; A: aneurisma; VI: ventrículo izquierdo; M: masculino; F: femenino; EV: extrasístole ventricular. 
Figura 1. Aneurisma en el ápex del VI (caso 5). Ecocardiografía, ventana sub costal, 4 cámaras.

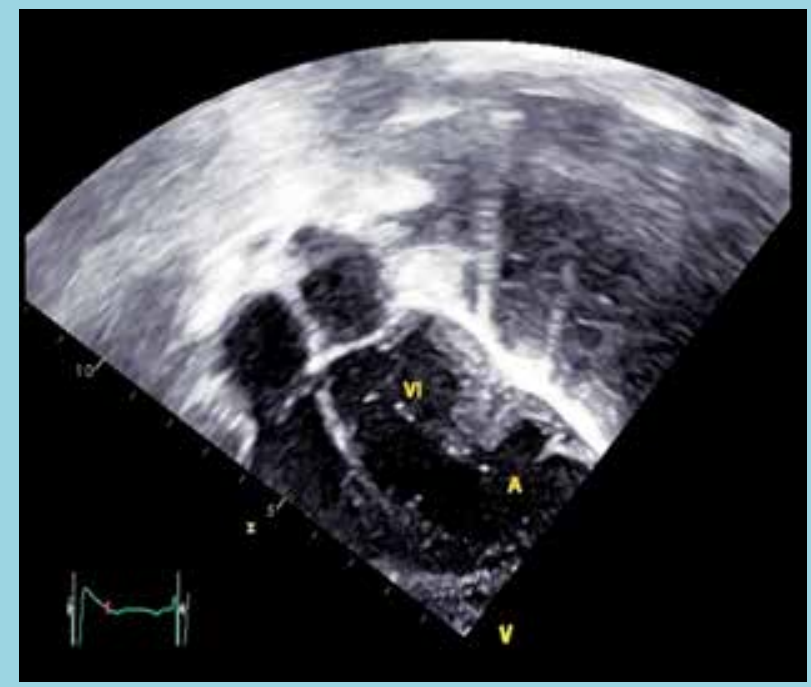

VI: ventrículo izquierdo, A: aneurisma.

Figura 2. Divertículo subtricuspídeo del VD (caso 1). Ecocardiograma 3D fetal.

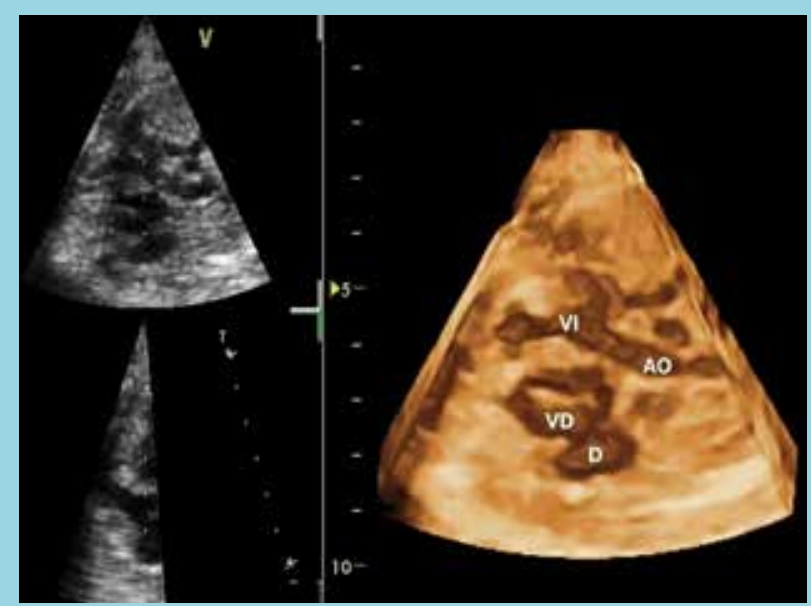

VD: ventrículo derecho, D: divertículo, VI: ventrículo izquierdo, AO: aorta

sentación fue por ecocardiografía fetal en dos pacientes $\mathrm{y}$ en los restantes por hallazgo de soplo cardíaco que fueron producto de comunicaciones interventriculares en dos, y de soplo funcional en un paciente.

El tipo de malformación, ubicación, aspectos de la radiografía de tórax, ECG y Holter de arritmias de 24 horas se detallan en la Tabla 1 .
Figura 3. Ecocardiografía Doppler tisular y $Q$ análisis, postnatal, (caso 1). Sincronía de la pared del divertículo subtricuspídeo (amarillo), pared libre del VD (rojo) y septum (verde).

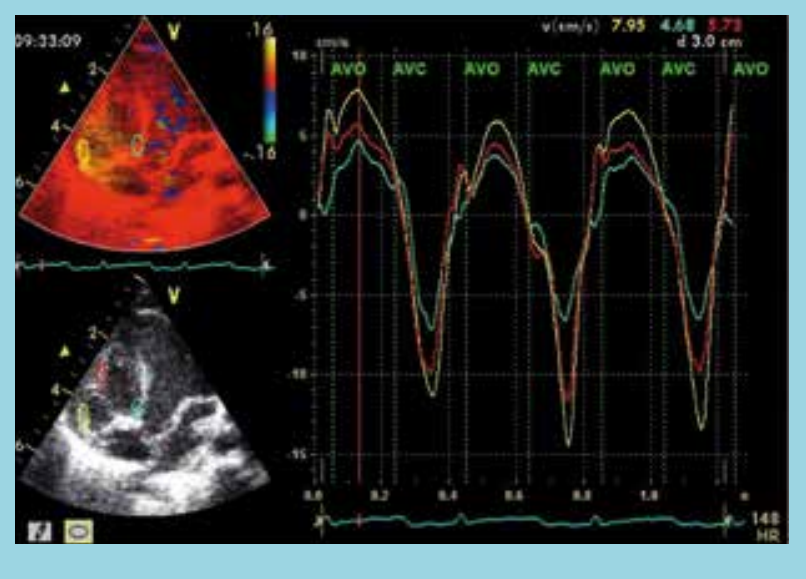

Dos aneurismas se ubicaron en el septum y dos en el ápex del ventrículo izquierdo (VI), como se observa en la Figura 1. El divertículo se localizó en la región subtricuspídea del ventrículo derecho (VD). No se visualizaron trombos intracardíacos. La función ventricular fue normal en todos los pacientes.

La ecocardiografía 3D (Figura 2) se utilizó en un paciente y permitió una mejor identificación de la anatomía del divertículo. En este paciente, además, se utilizó, Doppler tisular con $\mathrm{Q}$ análisis que confirmó la sincronía de la pared del divertículo con el resto de las paredes cardíacas (Figura 3).

Dos pacientes, ambos con aneurismas, presentaron comunicaciones interventriculares, perimembranosa en un paciente y musculares múltiples en el otro.

En tres pacientes se realizó RMC que confirmó el diagnóstico ecocardiográfico.

El seguimiento promedio fue de 48 meses (rango de 5 meses a 114 meses). Durante la evolución no se observaron cambios morfológicos significativos de las malformaciones. A los 9 años de seguimiento un paciente con aneurisma del septum presentó un episodio asintomático de taquicardia ventricular no sostenida que se trató con atenolol. Todos los pacientes recibieron aspirina profiláctica en dosis antiplaquetaria. No hubo fallecidos. El seguimiento en cuanto a tiempo y aparición de complicaciones se describe en la Tabla 2.

\section{Discusión}

Los aneurismas y divertículos cardíacos constituyen una 


\begin{tabular}{|c|c|c|c|c|}
\hline \multicolumn{3}{|c|}{ Tabla 2. Tiempo de seguimiento y presencia de eventos agregados. } \\
\hline N ${ }^{\circ}$ de Caso & $\begin{array}{c}\text { Tiempo } \\
\text { de seguimiento }\end{array}$ & Eventos & $\begin{array}{c}\text { Cambio en } \\
\text { la terapia }\end{array}$ & Fallecido \\
\hline 1 & 62 meses & No & No & No \\
\hline 2 & 37 meses & No & No & No \\
\hline 3 & 114 meses & TV no sostenida & Atenolol & No \\
\hline 4 & 25 meses & No & No & No \\
\hline 5 & 5 meses & No & No & No \\
\hline TV: taquicardia ventricular. & & & \\
\hline
\end{tabular}

malformación congénita infrecuente de la pared cardíaca1, 2. No se dispone de información epidemiológica con estudios prospectivos. Mayer ${ }^{3}$, en su serie retrospectiva de 43.000 ecocardiografías de pacientes adultos describió una prevalencia de $0,04 \%$. Albrecht ${ }^{4}$, en su estudio de 12.924 autopsias encontró una prevalencia de $0,02 \%$. Ohlow ${ }^{5}$ realizó una revisión bibliográfica de lo publicado en esta patología entre los años 1816 y 2014 encontrando 664 pacientes. Este autor estudió específicamente los de diagnóstico prenatal, que correspondieron a 42 pacientes (62\% aneurismas y $38 \%$ divertículos).

Los aneurismas y divertículos cardíacos pueden ubicarse en cualquiera de las cámaras cardíacas. El ventrículo izquierdo es la localización más frecuente, específicamente el ápex y el área perivalvular mitral ${ }^{6}$. En nuestra serie, 2 pacientes presentaron compromiso del ápex del VI, 2 pacientes del septum y 1 del VD.

Se asocian frecuentemente con malformaciones cardíacas y extracardíacas más frecuentemente defectos interventriculares. En una revisión de 411 casos de la literatura $108(26 \%)$, se asociaron con comunicación interventricular (CIV $)^{6}$. En nuestro grupo dos pacientes presentaron dicha asociación. Con respecto a las manifestaciones clínicas, existe un amplio espectro de presentación, desde pacientes asintomáticos (más frecuentes en la edad pediátrica), hallazgo de soplo cardíaco, precordalgia, disnea con ejercicio (descritos en aneurismas de localización submitral), arritmias, embolias, rotura y muerte súbita ${ }^{6-8}$. La presencia de soplo (3/5) fue la manifestación más frecuente en esta serie pediátrica. La edad de diagnóstico por lo general es tardía, en promedio $42 \pm 13$ años (rango 16 a 72 años) debido a que en la mayoría de las casos, se trata de hallazgos incidentales $^{8}$. Sin embargo, con la incorporación de la ecografía prenatal se ha adelantado la edad del diagnóstico. En nuestra serie dos pacientes fueron de pesquisa prenatal y la edad de diagnóstico promedio de la serie fue 13 meses.

Las alteraciones del ritmo pueden ser diversas, si bien la asociación etiológica no es clara ${ }^{9-12}$. Los hallazgos en el electrocardiograma van desde un trazado normal, presencia de extrasístoles ventriculares, bloqueo completo de rama derecha, taquicardia ventricular y signos de isquemia miocárdica. En esta serie dos pacientes presentaron alteraciones inespecíficas de la conducción intraventricular. El Holter de arritmias de 24 detectó la presencia de extrasistolía ventricular escasa en un paciente y en otro, durante el seguimiento, un episodio asintomático de taquicardia ventricular no sostenida.

La radiografía de tórax es un método poco sensible: sólo un pequeño número de pacientes presenta alteración en la morfología de la silueta cardíaca y/o cardiomegalia. La ecocardiografía Doppler color permite realizar el diagnóstico, como también detectar presencia de malformaciones asociadas. Las técnicas 3D y Doppler tisular (Q análisis) aportarán información sobre la calidad de la contracción de la fibra miocárdica, ayudando a definir si se trata de aneurismas o divertículos ${ }^{9-14}$. Estudios imagenológicos complementarios como RMC y Tomografía computada cardíaca pueden ser útiles en el estudio, pero no son exámenes de rutina en esta patología $^{12-14}$. En esta serie se realizó RMC en tres pacientes que no aportó información adicional.

Al no disponerse de grandes series de pacientes, con seguimientos de larga duración, las recomendaciones terapéuticas son difíciles de establecer, no existiendo estudios randomizados que avalen una determinada conducta. El tratamiento médico conservador incluye el uso de terapia antiplaquetaria y en algunos casos 
anticoagulación oral y antiarrítmicos, junto con seguimiento estricto para la detección precoz de complicaciones $^{13}$. La periodicidad del seguimiento y la duración de la terapia antiplaquetaria o anticoagulante no están definidas. El uso de anticoagulación oral es recomendado en pacientes con aneurismas grandes aquinéticos o posterior a embolia sistémica.

El manejo quirúrgico se ha indicado incluso en pacientes asintomáticos, como prevención de complicaciones potencialmente letales. En general, se indica en aque- llos defectos de gran tamaño, o de crecimiento progresivo, o en presencia de complicaciones como embolias, rotura o arritmias intratables con riesgo vital ${ }^{14-16}$.

Este estudio constituye la primera serie de pacientes pediátricos descrita en nuestro país con estas malformaciones. Si bien tiene las limitaciones de ser un estudio retrospectivo con escaso número de pacientes, aporta aspectos clínicos, de laboratorio y de seguimiento que contribuyen en el diagnóstico y su manejo en la edad pediátrica.

\section{Referencias}

1. GIANNOGLOU G, DELIOGLANIS S, PARASKEVAIDIS S, CHATZIZISIS Y, PARCHARIDIS G. Ventricular diverticulum: Definition, pathophysiology, clinical manifestations and treatment. Aristotle University Medical Journal 2007; 34:87-92.

2. MARIJON E, OU P, FERMONT L, CONCORDET S, LE BIDOIS J, SIDI D, et al. Diagnosis and outcome in congenital ventricular diverticulum and aneurysm. J Thorac Cardiovasc Surg 2006; 131: 433-437.

3. MAYER K, CANDINAS R, RADOUNLIS C, JENNI R. Kongenitale linksventrikuläre Aneurysmen und Divertikel: Klinik, Diagnostik und Verlauf. Schweiz Med Wochenschr 1999; 129: 1249-1256.

4. ALBRECHT G. Beitrag zur Morphologie und formalin Genese kongenitaler Divertikel. Zentralbl Allg Pathol 1972; 116: 42-47.

5. OHLOW MA, BRUNELLI M, LAUER B. Characteristics and outcome of primary congenital left ventricular aneurysm and diverticulum: analysis of cases from the literature. Prenatal Diagnosis 2014; 34: 1-7.

6. OHLOW MA. Congenital left ventricular aneurysms and diverticula: definition, pathophysiology, clinical relevance and treatment. Cardiology 2006; 106: 63-72.

7. OHLOW MA, SECKNUS MA, GELLER JC, VON KORN H, LAUER B. Kongenitale linksventrikuläre Aneurysmata und Divertikel des Erwachsenen. Pathophysiologie, klinische Präsentation und Therapieoptionen. Med Klin 2007; 102: $358-65$.

8. BRACHLOW A, SABLE C, SMITH S, SLACK M, MARTIN G. Fetal diagnosis and postnatal follow- up of an asymptomatic congenital left ventricular diverticulum. Pediatric Cardiology 2002; 23: 658-660.
9. OHLOW MA, LAUER B, GELLER JC. Prevalence and spectrum of abnormal electrocardiograms in patients with an isolated congenital left ventricular aneurysm or diverticulum. Europace 2009; 11: 1689-95.

10. ZHANG YL, SHEN E, WU ZH, XUE XP, ZHANG WX. Echocardiographic Findings on Congenital Left Ventricular Diverticulum and Aneurysm: Two Rare Cases and Literature Review. Echocardiography 2010; 27: 344-347.

11. ROMAGNOLI A, RICCI A, MOROSETTI D, FUSCO A, CITRARO D, SIMONETTI G ET AL. Congenital left ventricular diverticulum: Multimodality imaging evaluation and literature review J Saudi Heart Assoc 2015; 27: 61-67.

12. MCMAHON CJ, MONIOTTE S, POWELL AJ, DEL NIDO P, GEVA T. Usefulness of Magnetic Resonance Imaging Evaluation of Congenital Left Ventricular Aneurysms. Am J Cardiol 2007; 100: 310-315.

13. HAMAOKA K, ONAKA M, TANAKA T, ONOUCHI Z. Congenital ventricular aneurysm and diverticulum in children. Pediatr Cardiol. 1987; 8: 169- 75.

14. WANG W. ZHU W, WANG Y, LI J, GONG F. Congenital Left Ventricular Diverticulum Manifested as T-Wave Inversion in a Child. Pediatr Cardiol. 2010; 31: 881-883.

15. OKEREKE OU, COOLEY DA, FRAZIER OH. Congenital diverticulum of the ventricle. J Thorac Cardiovasc Surg. 1986; 91: 208-14.

16. DIBARDINO DJ, CHARAF-EDDINE A, GAYMES C, SHETH M, RIVARD A, SALAZAR JD. Left Ventricular Diverticulum: How We Do It. World Journal for Pediatric and Congenital Heart Surgery. 2013; 4: 318-321. 